Control of the wetting properties of an AISI 316L stainless steel surface by femtosecond laserinduced surface modification

This article has been downloaded from IOPscience. Please scroll down to see the full text article.

2012 J. Micromech. Microeng. 22105019

(http://iopscience.iop.org/0960-1317/22/10/105019)

View the table of contents for this issue, or go to the journal homepage for more

Download details:

IP Address: 141.211.173.82

The article was downloaded on 25/06/2013 at 20:34

Please note that terms and conditions apply. 


\title{
Control of the wetting properties of an AISI 316L stainless steel surface by femtosecond laser-induced surface modification
}

\author{
D H Kam, S Bhattacharya and J Mazumder ${ }^{1}$ \\ Center for Lasers and Plasmas in Advanced Manufacturing, University of Michigan, Ann Arbor, \\ MI 48109, USA \\ E-mail: mazumder@umich.edu
}

Received 13 October 2011, in final form 31 July 2012

Published 28 August 2012

Online at stacks.iop.org/JMM/22/105019

\begin{abstract}
A simple and effective method without vacuum to control the wetting properties of AISI 316L stainless steel using femtosecond laser pulses at high repetition rate has been developed. Both hydrophilic and hydrophobic surfaces were formed by creating micro-conical structures on the surface with femtosecond laser irradiation in air. The scan speed was found to be an effective parameter in controlling micro-cone morphology, size and number densities and contact angles during surface wettability experiments. It was found during surface wettability experiments that the contact angle of water varied from $0^{\circ}$ (superhydrophilic) to $113^{\circ}$ on laser micro-cone textured surfaces depending on processing conditions. Additionally, a superhydrophobic AISI 316L stainless steel surface was created (contact angle $\sim 150^{\circ}$ ) with a functionalized silane coating on already hydrophobic surface geometry.
\end{abstract}

(Some figures may appear in colour only in the online journal)

\section{Introduction}

In femtosecond (fs) laser irradiation, formation of conical micro-spikes has been observed on semiconductors [1-3] and metal surfaces $[4,5]$. The resulting conical textures have received enormous attention for their scientific significance and potential applications. One attractive application of such surfaces is their use in controlling surface wettability. Such wetting controllability has a wide range of applications including enhancement of heat transfer [6-8], adhesion improvement for surface coatings $[9,10]$ and self-cleaning and anti-icing surface fabrications [11, 12]. The roughness imposed by conical microstructures formed when irradiated with fs pulses can change wetted surface fraction so that the contact angle of liquids can be controlled [13, 14]. Although hydrophilic surfaces are equally important, previous studies on fs surface modifications were only focused on realization

\footnotetext{
1 Author to whom any correspondence should be addressed.
}

of hydrophobic surfaces. However, in the current investigation both hydrophobic and hydrophilic surfaces were fabricated via micro-cones formation by fs laser pulses at high repetition on AISI 316L stainless steel (SS) surfaces.

The ability to control the wetting properties of SS is extremely beneficial as a common metal in many areas of daily life as well as for scientific and industrial applications. Various existing methods have been employed to control the wetting properties of SS surfaces for preparing (a) hydrophilic surfaces via hydrophilic coatings [6,7], plasma treatments [8,9] and chemical treatments $[9,10]$ and (b) hydrophobic surfaces via hydrophobic coatings [12, 15-17]. However, these methods are generally expensive, complicated and time consuming. Some simple methods, such as chemical treatments, and cheap coatings are generally either evanescent or fragile. Moreover, no single method can cover the wide range of contact angles from the superhydrophilic to superhydrophobic condition. As mentioned earlier, in the current investigation a simple and reliable method to control the wettability of SS surfaces was developed. 


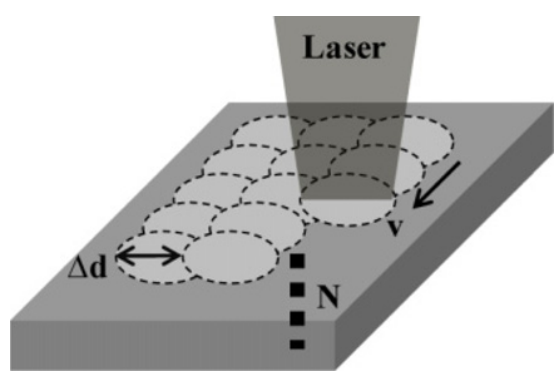

Figure 1. Schematic of the layer-by-layer ablation process, each layer being scanned parallel with a pitch $(\Delta d)$ of $10 \mu \mathrm{m}(N$ is the number of laser scanned layers and $v$ is the scan speed).

In this investigation two different types of micro-cone formations on AISI 316L SS surfaces under fs laser irradiation at high repetition rate $(\geqslant 500 \mathrm{kHz})$ were demonstrated. It was observed that these two types of micro-cones were generated depending on the pulse fluence, i.e. low (near threshold to $\left.\sim 0.26 \mathrm{~J} \mathrm{~cm}^{-2}\right)$ and high fluence $\left(>\sim 0.50 \mathrm{~J} \mathrm{~cm}^{-2}\right)$. For both formation processes, by changing the scanning speed $v$, average micro-cone size (or micro-cone number density) was controlled. The influence of these two different surfaces on wetting properties was investigated. It was observed that these micro-cones created both hydrophilic and hydrophobic surfaces on AISI 316L SS. Contact angles were tuned between $0^{\circ}$ and near $120^{\circ}$. Also, it was demonstrated that superhydrophobic surfaces could be created by additional hydrophobic coatings on micro-conical textured surfaces.

\section{Experimental details}

AISI 316L SS experimental samples were prepared for laser irradiation by mechanical polishing followed by methanol cleaning. The irradiating laser used was an fs-pulsed fiber laser with a wavelength of $1043 \mathrm{~nm}$ that delivered $\sim 490 \mathrm{fs}$ laser pulses with $\sim 10 \mu \mathrm{J}$ pulse energy. The pulse energy was varied using a combination of a half-wave plate and a polarizer. The beam shape was found to be near Gaussian. Using a $167 \mathrm{~mm}$ focal length $F$-theta lens spot size, of full width at $1 / \mathrm{e}^{2}$ maximum, at focus was $D \sim 50 \mu \mathrm{m}$ in diameter. A twomirror galvanometric scanner manufactured by SCANLAB, Germany (model: hurrySCAN ${ }^{\circledR}$ II 14 , maximum wavelength $1064 \mathrm{~nm}$ ) and a Z-direction stage were used to control the motion of laser beam spots and z-position (fixed at focus). The beam was linearly polarized perpendicular to scan directions. In the layer-by-layer ablation process as shown in the schematic (figure 1), each layer was scanned with multiple parallel lines with a center-to-center pitch $(\Delta d)$ of $10 \mu \mathrm{m}$. The number of layers $(N)$ and the scanning speed $(v)$ were manipulated to form micro-cones. The threshold fluence for SS for micro-cone formation was found to be $F_{\text {th }} \sim 0.109$ $\mathrm{J} \mathrm{cm}{ }^{-2}$. Experiments were performed in air with both 500 and $1000 \mathrm{kHz}$ repetition rates. Micro-cone number density variation was found to be larger in the case of high-fluence conditions $\left(F \sim 1.014 \mathrm{~J} \mathrm{~cm}^{-2}\right.$ at $\left.1000 \mathrm{kHz}\right)$ compared to lowfluence conditions $\left(F \sim 0.253 \mathrm{~J} \mathrm{~cm}^{-2}\right.$ at $\left.500 \mathrm{kHz}\right)$ for $v$ ranging from 120 to $2400 \mathrm{~mm} \mathrm{~s}^{-1}$. Following experiments irradiated surface microstructures were examined by scanning electron microscopy (SEM) (Philips ${ }^{\mathrm{TM}}$ XL30 FEG scanning electron microscope). Each SEM micrograph was taken at a $45^{\circ}$ angle to the surface. $\operatorname{EDAX}^{\circledR}$ analyses of experimental sample surfaces were performed to determine chemical composition of microstructural features.

Surface wettability tests were performed on irradiated and microstructured AISI 316L SS sample surfaces with $3 \mu \mathrm{L}$ deionized (DI) water droplets, and static contact angles were measured by the sessile drop method. Additionally, to achieve further hydrophobicity or superhydrophobicity, textured surfaces with micro-cones formed under high-fluence conditions were coated with a functionalized silane (tridecafluoro-1,1,2,2,-tetrahydrooctyl1-trichlorosilane) by the chemical vapor diposition (CVD) process, and similar surface wetability tests were performed. Contact angles of water droplets were measured from photographs taken during experiments with a high-speed camera. Each contact angle value was an average of three experimental measurements.

\section{Results and discussion}

In the current investigation, fs laser ablation at high repetition rates of 500 and $1000 \mathrm{kHz}$ was used to systematically develop micro-cone morphologies in air on AISI 316L SS surfaces, through progressive evolution at different laser fluences for a wide range of scanning velocities. Two types of micro-cone morphologies formed at these high repetition rates under highand low-fluence conditions were clearly distinguished. It was observed that the micro-cone formation, i.e. the nucleation and growth process, is governed by a dynamic balance between redeposition and ablation processes. This is evident from dendritic microstructure observed during micro-cones crosssection analysis presented elsewhere [18]. Initially, surface is roughened by fs pulses, and then non-uniformity results in the local variation of absorption leading to evolution of micro-cone arrays. At low fluence $\left(F \sim 0.253 \mathrm{~J} \mathrm{~cm}^{-2}\right)$, due to low energy density per pulse, inclined walls of micro-cones did not ablate because the irradiation fluence required for ablation increased due to the increased effective surface area, and redeposition of ablated materials dominated. This led to preferential material removal as shown in figure $2(b)$. The selectivity by threshold shift is critical when the fluence is near the ablation threshold value. At high fluence $\left(F \sim 1.014 \mathrm{~J} \mathrm{~cm}^{-2}\right.$ ), due to energy density being far beyond ablation threshold, extra energy enables an overall material removal and causes the ablation process to dominate over the redeposition process. Higher absorptance in valleys induced by laser radiation and surface wave interference causes higher but uniform material removal rate from both inclines and peaks. It was observed during experiments that the aspect ratio (height to width) of microcones increased with increasing number of scanned layers. Compared to micro-cones formed by low fluence, cones formed at high fluence have a relatively uniform distribution and higher aspect ratio. For both micro-cone morphologies, surface morphology did not change with increasing $N$ once the conical structures were fully developed. $N$ was increased up to the saturation points in both micro-cone formations. 


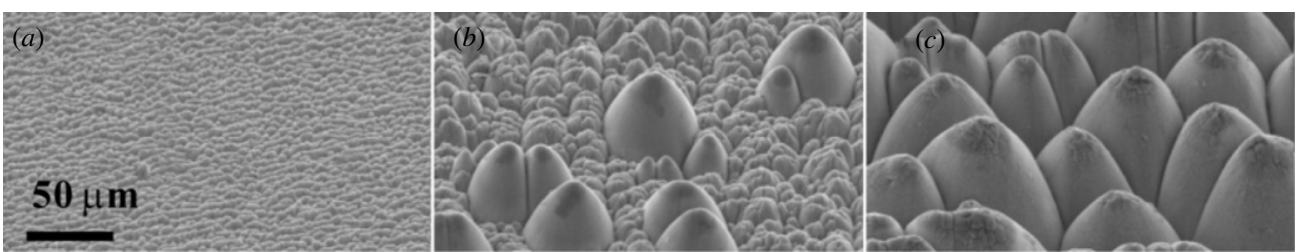

Figure 2. SEM micrographs of the 316L SS surface after $(a) N=100,(b) N=800$ and $(c) N=3000$. Pulses with $F \sim 0.253 \mathrm{~J} \mathrm{~cm}^{-2}(2 \mu \mathrm{J}$ at focus) at $1000 \mathrm{kHz}$ scanned with $v=2400 \mathrm{~mm} \mathrm{~s}^{-1}$. Each SEM micrograph was taken at a $45^{\circ}$ angle to the surface.

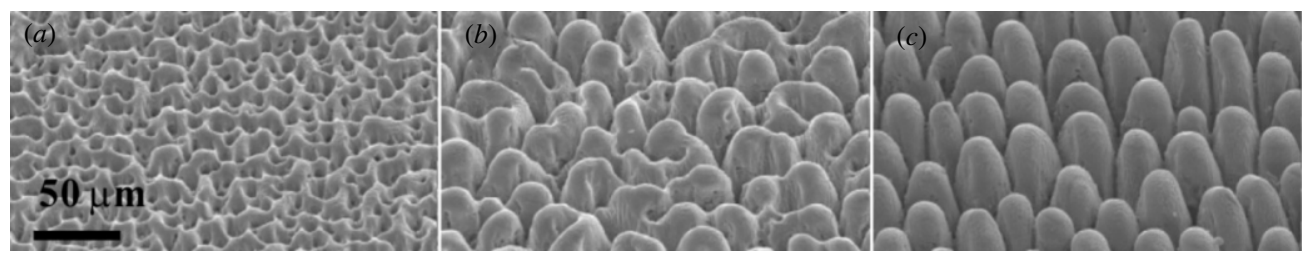

Figure 3. SEM micrographs of the 316L SS surface after $(a) N=8,(b) N=40$ and $(c) N=300$. Laser pulses with $F \sim 1.014 \mathrm{~J} \mathrm{~cm}^{-2}(8 \mu \mathrm{J}$ at focus) at $1000 \mathrm{kHz}$ were scanned with $v=600 \mathrm{~mm} \mathrm{~s}^{-1}$. Each SEM micrograph was taken at a $45^{\circ}$ angle to the surface.

Table 1. Average ablation depths and ablation rates for low-fluence condition specimens (figure 2).

\begin{tabular}{lrll}
\hline Figure 2 & $N$ & $\begin{array}{l}\text { Average ablation } \\
\text { depth }(\mu \mathrm{m})\end{array}$ & $\begin{array}{l}\text { Ablation rate } \\
\left(\mathrm{mm}^{3} \mathrm{~min}^{-1}\right)\end{array}$ \\
\hline$(a)$ & 100 & 06.46 & 0.068 \\
$(b)$ & 800 & 69.6 & 0.125 \\
$(c)$ & 3000 & 52.2 & 0.025 \\
\hline
\end{tabular}

Table 2. Average ablation depths and ablation rates for high-fluence condition specimens (figure 3).

\begin{tabular}{lrcl}
\hline Figure 3 & \multicolumn{1}{c}{$N$} & $\begin{array}{l}\text { Average ablation } \\
\text { depth }(\mu \mathrm{m})\end{array}$ & $\begin{array}{l}\text { Ablation rate } \\
\left(\mathrm{mm}^{3} \mathrm{~min}^{-1}\right)\end{array}$ \\
\hline$(a)$ & 8 & 2 & 0.089 \\
$(b)$ & 40 & 130 & 1.116 \\
$(c)$ & 300 & 592 & 0.624 \\
\hline
\end{tabular}

Tables 1 and 2 show the average ablation depths and ablation rates for corresponding specimens shown in figures 2 and 3 , respectively. It was observed that in both tables 1 and 2 ablation rate increased from $(a)$ to $(b)$ and then decreased to (c). However, under the high-fluence condition (table 2) this decrease was less as compared to that under the low-fluence condition (table 1). Also, it was observed that under the highfluence condition ablation depth increased with increasing $N$ whereas under the low-fluence condition it decreased after reaching a maximum depth.

Figure 4 shows SEM micrographs of sample surfaces formed by low fluence $\left(F \sim 0.180 \mathrm{~J} \mathrm{~cm}^{-2}\right)$ at various scan speeds $(v)$ and photographs of water droplets on corresponding surfaces. The number density of cones increases (or the distance between cones decreases) with increasing $v$. As $v$ increases, thermal stresses induced by heat accumulation decreases [20]. However, when $v$ is increased further $\left(>1200 \mathrm{~mm} \mathrm{~s}^{-1}\right.$ ) micro-cones disappeared forming a smooth surface as shown in figure 4(c). For these samples, surfaces were all hydrophilic, $\theta<30^{\circ}$, while surfaces scanned at $v>480 \mathrm{~mm} \mathrm{~s}^{-1}$ are superhydrophilic $\left(\theta=0^{\circ}\right)$. The surface wettability was determined by the combined effect of the surface chemistry and geometry of surface microstructure. For homogeneous hydrophilic surfaces, the Wenzel model is generally used to relate the Wenzel apparent contact angle, $\theta_{w}$, with the Young contact angle, $\theta_{Y}$, and the roughness ratio, $r$. The roughness ratio was defined as the ratio of the actual area of the solid surface to its geometric surface [21],

$$
\cos \theta_{w}=r \cos \theta_{Y} \text {. }
$$

According to equation (1), if roughness due to micro-cones has more dominant influence on contact angles, $\theta_{Y}$ in figure 4(a) must be less than that in figure 4(c) because $r>1$. But the current observation shows the reverse trend. Consequently, the surface geometry dependence of the contact angle was negligible for hydrophilic surfaces indicating a strong surface chemistry dependence. In $\mathrm{EDAX}^{\circledR}$ analysis, it was found that micro-cone textured surfaces formed with low-fluence irradiation had approximately 3.2 to 4.9 times (weight base) more oxide than that of the polished surface. Oxides were believed to have chemically altered the hydrophilicity of surfaces due to their strong affinity for hydroxylation [22].

For samples prepared by high fluence (figure 5), microcone number density also increased with $v$. Although this trend is the same as observed in the case of low fluence, a different explanation could be given for this behavior. Such a trend was caused since effective average fluence per scan layer decreased with increasing $v$. This decrease in average fluence reduced micro-cone number density similar to that found in previous studies by other researchers using fs pulses and high fluence $[2,4,5]$. For these samples, wide ranges of resulting contact angles were measured as shown in the plot in figure 6. For $v \leqslant 240 \mathrm{~mm} \mathrm{~s}^{-1}$, surfaces were superhydrophilic $\left(\theta \sim 0^{\circ}\right)$. At $v=480 \mathrm{~mm} \mathrm{~s}^{-1}$, water droplets formed oval shapes and so two contact angles, $\theta=54.8^{\circ}$ and $\theta=79.3^{\circ}$ for long and short axes, respectively, were measured. For $v>$ $600 \mathrm{~mm} \mathrm{~s}^{-1}$, surfaces became hydrophobic $\left(\theta>90^{\circ}\right)$ and the maximum contact angle for the speed range was measured as $\theta \sim 113^{\circ}$. Chemically the structured surface was hydrophilic because the surface layer had high amount of oxide content (approximately 2.8 to 5.8 times more than the polished surface 


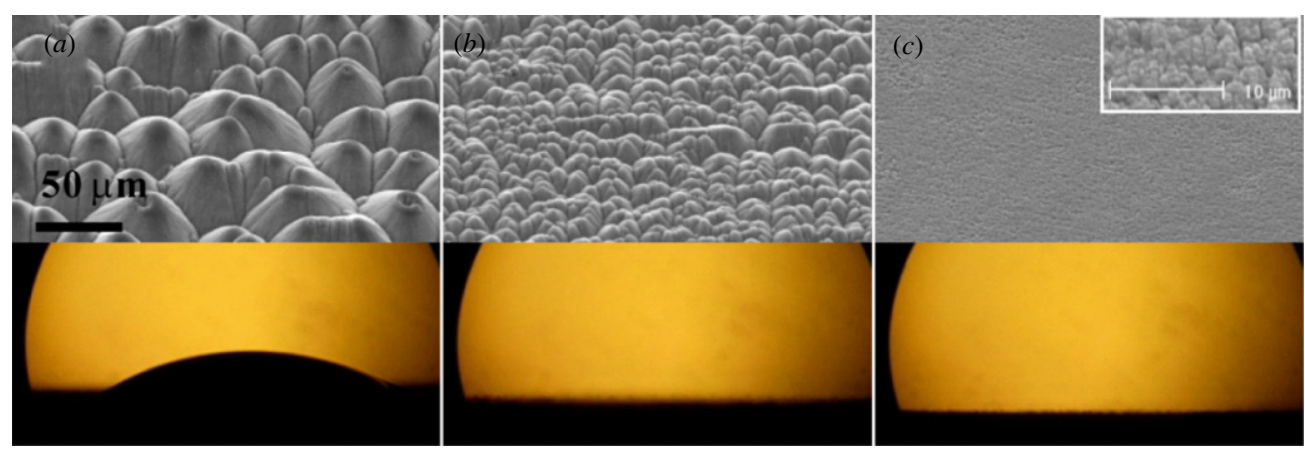

Figure 4. SEM micrographs of the AISI 316L SS surface after $N / v=1 \mathrm{~s} \mathrm{~mm}^{-1}$ and corresponding photographs of $3 \mu \mathrm{L}$ DI water droplets on these surfaces. Laser pulses with $F \sim 0.180 \mathrm{~J} \mathrm{~cm}^{-2}(7 \mu \mathrm{J}$ pulses at $3 \mathrm{~mm}$ defocused below surface) at $500 \mathrm{kHz}$ were used and samples were scanned with $v(a) 240 \mathrm{~mm} \mathrm{~s}^{-1},(b) 480 \mathrm{~mm} \mathrm{~s}^{-1}$ and (c) $2400 \mathrm{~mm} \mathrm{~s}^{-1}$. Each SEM micrograph was taken at a $45^{\circ}$ angle to the surface.
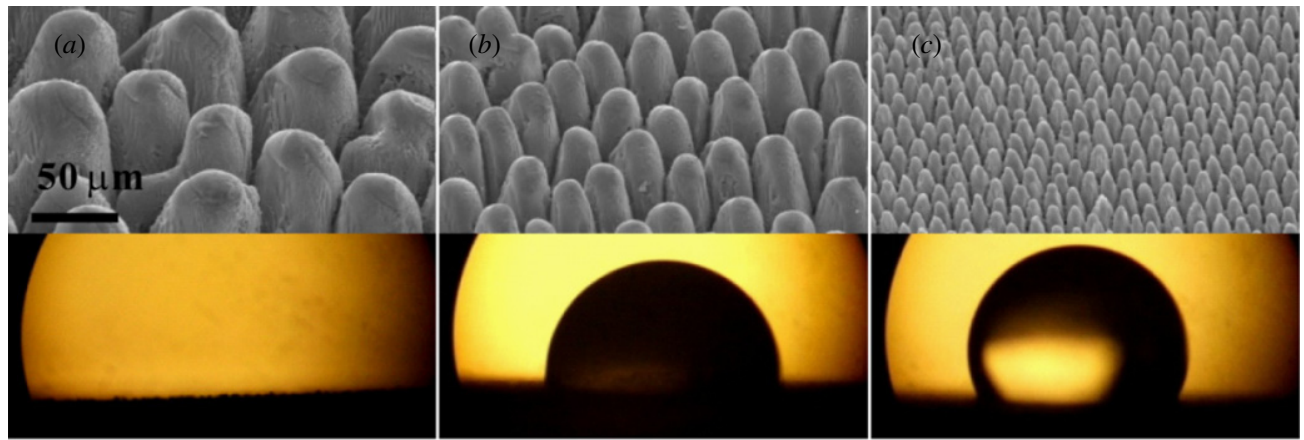

Figure 5. SEM micrographs of the $316 \mathrm{~L} \mathrm{SS}$ surface after $N / v=0.5 \mathrm{~s} \mathrm{~mm}^{-1}$ and corresponding photographs of $3 \mu \mathrm{L}$ water droplets on surfaces. Laser pulses with $F \sim 1.014 \mathrm{~J} \mathrm{~cm}^{-2}(8 \mu \mathrm{J}$ pulses at focus) at $1000 \mathrm{kHz}$ were used and samples were scanned with $v$ (a) $240 \mathrm{~mm} \mathrm{~s}^{-1}$, (b) $600 \mathrm{~mm} \mathrm{~s}^{-1}$ and (b) $2400 \mathrm{~mm} \mathrm{~s}^{-1}$. Each SEM micrograph was taken at a $45^{\circ}$ angle to the surface.

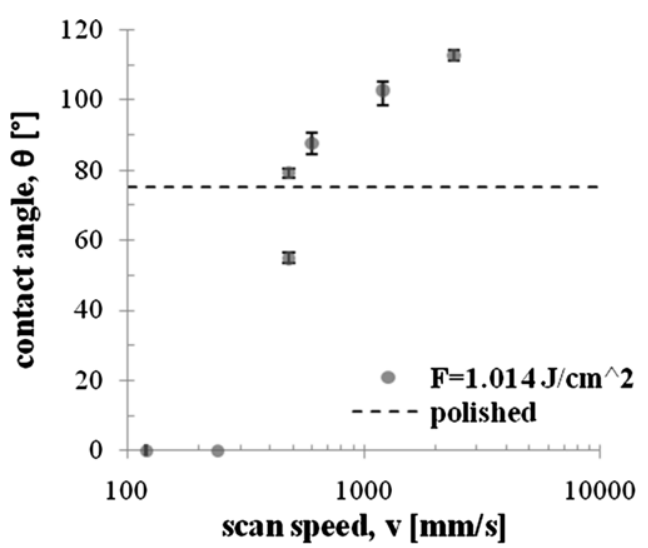

Figure 6. Plot of static contact angles $(\theta)$ of $3 \mu \mathrm{L}$ of the DI water droplet on microstructured SS surfaces as a function of scan speed $(v)$. The microstructured surfaces were prepared with a fluence of $F=1.014 \mathrm{~J} \mathrm{~cm}^{-2}$. The dashed line gives a reference contact angle $\left(\sim 75^{\circ}\right)$ of the mechanically polished surface.

in weight base) similar to low-fluence processes. Contrary to microstructured surfaces formed at low fluence, liquid contact angles for these surfaces strongly depended on the surface geometry. This is attributed to the high aspect ratio of micro-cones which prevented full penetration of liquid into valleys between them. Therefore, resulting liquid contact angles were determined by competing effects of geometrical hydrophobicity and chemical hydrophilicity. Additionally, the dependence of contact angles on the micro-cone number density was contrary to observations in previous studies $[13,14]$. This is probably due to the difference in micro-cone sizes. Typically, for conical spikes on silicon surfaces formed by fs pulses in $\mathrm{SF}_{6}$, the distance between cones $(d)$ is less than $5 \mu \mathrm{m}[13,14]$. However, in the current investigation the distance between micro-cones ranged from 10 to $70 \mu \mathrm{m}$.

To achieve further hydrophobicity, microstructured surfaces formed by high fluence were coated with a functionalized silane, tridecafluoro-1,1,2,2,-tetrahydrooctyl1-trichlorosilane, via a CVD process. The resulting surfaces were superhydrophobic, as shown in figure 7, as it was difficult to detach the water droplet from the pipet tip. The water droplet did not attach itself to the surface until the volume of the droplet was increased to $9.7 \mu \mathrm{L}$. Thus, contact angles were measured for $9.7 \mu \mathrm{L}$ DI water droplets as a function of the scan speed as presented in figure 8. It was found that the contact angles showed negligible dependence on the scan speed and were maintained at approximately $150^{\circ}$ for all scan speeds. Considering the contact angle for the polished surface with silane coating $\left(\theta \sim 101^{\circ}\right)$, the hydrophobic surface microstructures increased contact angles by approximately $50^{\circ}$.

In a previously published work by Wu et al [19], authors formed 'conical-shaped spikes' on AISI 316L surfaces by fs laser ablation under vacuum, at a very low repetition rate of $1 \mathrm{kHz}$ and a single scanning velocity of $1 \mathrm{~mm} \mathrm{~s}^{-1}$. On the other hand, in the current investigation scanning velocity combined with high repetition rate was efficiently used to control 


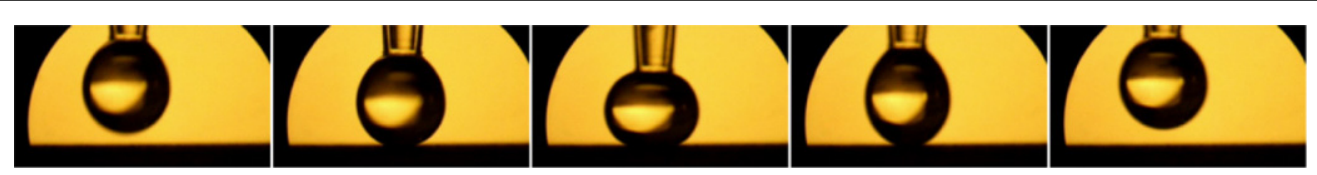

Figure 7. Progressive photographs of a $7 \mu \mathrm{L}$ DI water drop on the microstructured surface (see figure $5(a)$ for surface topology) with silane coating. Superhydrophobicity prevented the water droplet from attaching to the surface.

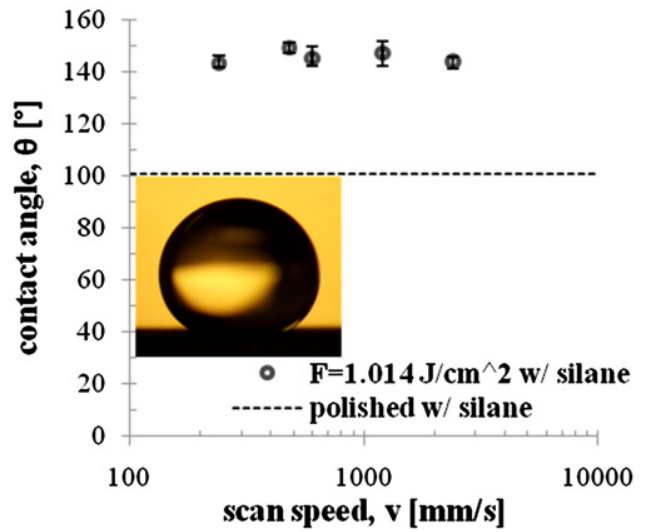

Figure 8. The contact angles, $\theta$, of DI water $(9.7 \mu \mathrm{L})$ on the microstructured SS surface with silane coating as a function of scan speed, $v$. The microstructures were prepared by the fluence of $F=$ $1.014 \mathrm{~J} \mathrm{~cm}^{-2}$. The dashed line gives a reference contact angle $\left(\sim 101^{\circ}\right)$ of the mechanically polished surface with silane coating.

micro-cone size and number density on AISI 316L surfaces. It was demonstrated that scanning speed, which is easily and precisely controlled in automated laser micromachining systems, is a key parameter to control the micro-cone surface morphology. Also, the laser pulse energy system used in the current investigation can be more easily automated as compared to the system used in the above-referenced article [19].

In the current investigation, all specimens were prepared in air (without vacuum) and the wetting properties of laser microstructured surfaces were characterized (i) without any additional surface treatment and additionally (ii) with silane coating treatment. On the other hand, the authors in the abovereferenced article [19] coated laser microstructured AISI 316L surfaces with silane for wettability tests under vacuum to show that the surfaces were superhydrophobic.

In the current investigation both hydrophilic and hydrophobic surfaces were created on the same AISI 316L surface, covering a wide range of contact angles from superhydrophilicity to superhydrophobicity, which has never been reported to have been accomplished in one single process. This was achieved, as mentioned above, by primarily varying scanning speed at high repetition rates. In all previous studies $[13,14,19]$, the fluence was used as the primary parameter to control the cone size and thus the surface wettability. Hence in the current investigation, it was shown that the scanning speed is another key parameter that can be controlled to achieve the same results. Moreover, laser scanning speed control is easier to be implemented in an automated system as compared to laser fluence control. Also current generations of fs lasers do not have enough pulse energy to cover the wide range of surface wettability conditions (hydrophilicity to hydrophobicity) presented in the current investigation. This makes the current approach very unique and distinguishes it from all earlier reported attempts. Overall this makes the current process very simple and effective for creating periodic micro-cones on alloy surfaces without vacuum to control surface wettability (hydrophilic and hydrophobic) in one single process.

The ability to switch between manufacturing selective wettability surfaces may be extremely beneficial in fabricating devices such as lab on a chip, sensors, medical instruments, etc. Superhydrophilicity is promising for improved heat transfer coefficients and adhesion of surface coatings. Superhydrophobicity may pave the way for cost-effective antifrosting and self-cleaning solutions for various applications.

\section{Conclusions}

A unique technique capable of manufacturing both hydrophilic and hydrophobic surface on stainless steel (SS) with relatively simple femtosecond laser treatment was demonstrated. Using low pulse energy $(<10 \mu \mathrm{J})$ and high repetition rate $(\geqslant 500 \mathrm{kHz})$, AISI $316 \mathrm{~L}$ SS surfaces were manipulated to control wettability which was demonstrated by a wide range of liquid contact angles. In addition to the fluence, the scan speed played a key role in controlling surface wettability by changing micro-cone number density (or the size of micro-cone microstructures). Furthermore, superhydrophobic surfaces were manufactured by coating functionalized silane via a CVD process on micro-cone textured AISI 316L SS surfaces.

\section{Acknowledgments}

The research work was financially sponsored by NSF IUCRC under the program 'Lasers and Plasmas in Advanced Manufacturing'. The authors would like to acknowledge Dr Sung-Jin Kim at the University of Michigan, Ann Arbor, for his help in liquid contact angle measurements.

\section{References}

[1] Her T-H, Finlay R J, Wu C, Deliwala S and Mazur E 1998 Microstructuring of silicon with femtosecond laser pulses Appl. Phys. Lett. 73 1673-5

[2] Her T-H, Finlay R J, Wu C and Mazur E 2000 Femtosecond laser-induced formation of spikes on silicon Appl. Phys. A $70383-5$

[3] Bones J, Baudach S, Krüger J, Kautek W and Lenzner M 2002 Femtosecond laser ablation of silicon-modification thresholds and morphology Appl. Phys. A 74 19-25

[4] Nayak B K, Gupta M C and Kolasinski K W 2008 Formation of nano-textured conical microstructures in titanium metal 
surface by femtosecond laser irradiation Appl. Phys. A 90 399-402

[5] Nayak B K and Gupta M C 2010 Self-organized micro/nano structures in metal surfaces by ultrafast laser irradiation Opt. Lasers Eng. 48 940-9

[6] McDonnell A M P, Beving D, Wang A, Chen W and Yan Y 2005 Hydrophilic and antimicrobial zeolite coatings for gravity-independent water separation $A d v$. Funct. Mater. $15336-40$

[7] Liu J, Aguilar G, Munoz R and Yan Y 2008 Hydrophilic zeolite coatings for improved heat transfer: a quantitative analysis AIChE J. 54 779-90

[8] Takata Y, Hidaka S, Yamashita A and Yamamoto H 2004 Evaporation of water drop on a plasma-irradiated hydrophilic surface Int. J. Heat Fluid Flow 25 320-8

[9] Niemi R et al 2010 Surface pretreatment of austenitic stainless steel and copper by chemical, plasma electrolytic or $\mathrm{CO}_{2}$ cryoblasting techniques for sol-gel coating Surf. Coat. Technol. 204 2424-31

[10] Lattner D and Jennissen H P 2009 Preparation and properties of ultra-hydrophilic surfaces on titanium and steel Mater.wiss. Werkst.tech. 40 108-16

[11] Patankar N 2004 Mimicking the lotus effect: influence of double roughness structures and slender pillars Langmuir 20 8209-13

[12] Cao L, Jones A K, Sikka V K, Wu J and Gao D 2009 Anti-icing superhydrophobic coatings Langmuir 25 12444-8

[13] Zorba V, Persano L, Pisignano D, Athanassion A, Stratakis E, Cingolani R, Tzanetakis P and Fotakis C 2006 Making silicon hydrophobic: wettability control by two-length scale simultaneous patterning with femtosecond laser irradiation Nanotechnology 17 3234-8

[14] Baldacchini T, Carey J E, Zhou M and Mazur E 2006 Superhydrophobic surfaces prepared by microstructuring of silicon using a femtosecond laser Langmuir 22 4917-9

[15] Jagannathan R and Mehta R V 2006 Continuous, atmospheric process to create organic clusters and nanostructured, functional films Adv. Funct. Mater. 16 633-9

[16] Wang Q, Cui Z, Xiao Y and Chen Q 2007 Stable highly hydrophobic and oleophilic meshes for oil-water separation Appl. Surf. Sci. 253 9054-60

[17] Chen L J, Chen M, Zhou H D and Chen J M 2008 Preparation of super-hydrophobic surface on stainless steel Appl. Surf. Sci. 255 3459-62

[18] Bhattacharya S, Kam D H, Song L and Mazumder J 2012 Characterization of individual micro-needles formed on alloy surfaces by femtosecond laser ablation Metall. Mater. Trans. A 43 2574-80

[19] Wu B., Zhou M., Li J, Te X, Li G and Cai L 2009 Superhydrophobic surfaces fabricated by microstructuring of stainless steel using a femtosecond laser Appl. Surf. Sci. 256 61-6

[20] Eaton S M, Zhang H, Herman P R, Yoshino F, Shah L, Bovatsek J and Arai A Y 2005 Heat accumulation effects in femtosecond laser-written waveguides with variable repetition rate Opt. Express 13 4708-16

[21] Wenzel R N 1936 Resistance of solid surfaces to wetting water Ind. Eng. Chem. 28 988-94

[22] Gentleman M M and Ruud J A 2010 Role of hydroxyls in oxide wettability Langmuir 26 1408-11 